\title{
Dynamic Stark Control of Torsional Motion by a Pair of Laser Pulses
}

\author{
Lauge Christensen, Jens H. Nielsen, Christian B. Brandt, Christian B. Madsen, and Lars Bojer Madsen \\ Department of Physics and Astronomy, Aarhus University, Ny Munkegade 120, DK-8000 Aarhus C, Denmark \\ Craig S. Slater, Alexandra Lauer, and Mark Brouard \\ Department of Chemistry, University of Oxford, the Physical and Theoretical Chemistry Laboratory, \\ South Parks Road, Oxford OXI 3QZ, United Kingdom \\ Mikael P. Johansson \\ Department of Chemistry, Laboratory for Instruction in Swedish, University of Helsinki, A.I. Virtanens Plats 1, \\ P.O. Box 55, FI-00014 Helsinki, Finland \\ Benjamin Shepperson and Henrik Stapelfeldt \\ Department of Chemistry, Aarhus University, Langelandsgade 140, DK-8000 Aarhus C, Denmark
}

(Received 27 March 2014; published 12 August 2014)

\begin{abstract}
The torsional motion of a molecule composed of two substituted benzene rings, linked by a single bond, is coherently controlled by a pair of strong $\left(3 \times 10^{13} \mathrm{~W} \mathrm{~cm}^{-2}\right)$, nonresonant $(800 \mathrm{~nm}) 200$-fs-long laser pulses - both linearly polarized perpendicular to the single-bond axis. If the second pulse is sent at the time when the two benzene rings rotate toward (away from) each other the amplitude of the torsion is strongly enhanced (reduced). The torsional motion persists for more than $150 \mathrm{ps}$ corresponding to approximately 120 torsional oscillations. Our calculations show that the key to control is the strong transient modification of the natural torsional potential by the laser-induced dynamic Stark effect.
\end{abstract}

DOI: 10.1103/PhysRevLett.113.073005

PACS numbers: $33.15 . \mathrm{Hp}, 33.80 . \mathrm{Rv}, 42.50 . \mathrm{Hz}$

With their large bandwidth, highly configurable pulse shape [1] and polarization state [2], precise timing and high intensity, femtosecond (fs) laser pulses have been the central element in numerous coherent control schemes of chemical reactivity and intramolecular dynamics $[3,4]$. One approach to molecular control exploits the high intensity aspect of fs pulses to transiently exert forces on molecules, through the interaction between the electric field and the dipole it induces in a molecule. The use of this polarizability interaction to drive vibrations, rotations, or reactions in a desired direction has been termed dynamic Stark control (DSC) [5]. Its most widespread application is in laser-induced alignment of molecules - both in the impulsive [6] and the adiabatic limit [7] — but it has also been used to control vibrations of diatomic molecules [8,9], torsional motion of chiral molecules [10-13], data storage and readout in atoms [14], the velocity of molecules $[15,16]$, and the branching ratio of different final states in photodissociation of molecules [17].

A most attractive feature of DSC is that it acts on the nuclear motion of the molecules without inducing any electronic excitation; i.e., the molecule remains on the initial electronic potential surface. This is ensured by employing a nonresonant laser field such that no linear absorption takes place - in practice obtained by the use of near-infrared laser sources. Also, the laser pulse intensity is kept sufficiently low such that multiphoton ionization or dissociation is negligible. This restriction on the maximum intensity naturally implies a limitation to the degree of DSC by a single laser pulse. In the case of laser-induced alignment it was shown that this limitation can be compensated for by employing two or more optimally synchronized pulses to enhance the degree of alignment [18-21].

In the present work, we demonstrate experimentally and theoretically that two (or more) optimally timed laser pulses can also strongly improve the DSC efficiency when applied to induce torsion in a substituted biphenyl molecule, i.e., twisting of the two phenyl rings around the joining axis. This marks a significant step forward compared to previous works where only a single laser pulse was used to induce torsion $[10,11,13]$. Figure 1 shows the molecule studied, 3,5-difluoro-3', 5'-dibromo-4'-cyanobiphenyl, to be denoted FBCBP, and the dihedral angle, $\phi_{d}=\phi_{\mathrm{F}}-\phi_{\mathrm{Br}}$, the natural molecular coordinate characterizing torsion. Here $\phi_{\mathrm{F}}$ and $\phi_{\mathrm{Br}}$ denote the orientation of the $\mathrm{F}$ - and $\mathrm{Br}$-containing rings, respectively. In particular, we observe that the maximum amplitude of torsion around the equilibrium geometry can be enhanced from $\sim 3^{\circ}$ with one pulse to $\sim 6^{\circ}$ with two intense laser pulses, termed kick pulses, provided the second pulse arrives one torsional period after the first pulse, when $\phi_{d}$ is decreasing and the two rings rotate toward each other. If, instead, the second pulse is sent already after half a period, when $\phi_{d}$ is increasing, the torsional motion is essentially cancelled. Femtosecond timed Coulomb explosion combined with a novel correlation technique of the emission directions of the exploding fragment ions enable direct 


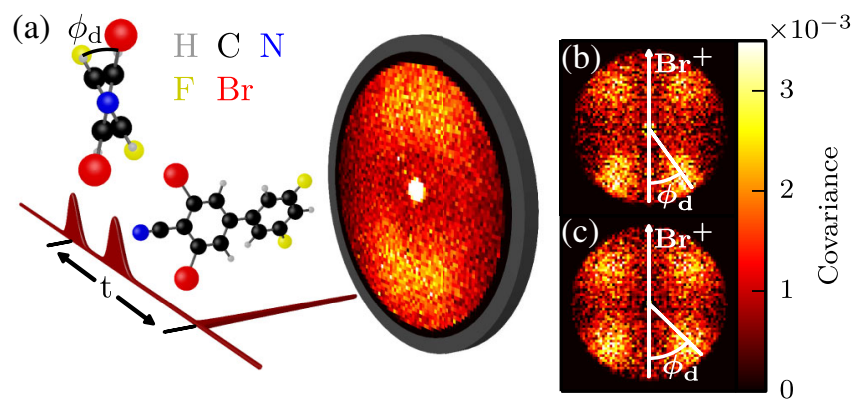

FIG. 1 (color online). (a) Schematic of the experiment showing the FBCBP molecule (atoms labeled by color), the dihedral angle $\phi_{d}$, the detector with a raw $\mathrm{F}^{+}$ion image, the two kick laser pulses (vertical polarization) that induce torsional motion and the probe laser pulse (horizontal polarization) that Coulomb explodes the molecules such that the instantaneous $\phi_{d}$ value can be determined from the $\mathrm{F}^{+}$and $\mathrm{Br}^{+}$ion images. A third laser pulse (not shown) keeps the molecule aligned as illustrated. Details are given in the text. (b),(c) Covariance images showing the emission direction of $\mathrm{F}^{+}$ions with respect to the $\mathrm{Br}^{+}$ion recoil (vertical). (b) is recorded at $t=4.00 \mathrm{ps}$ and is obtained from the $\mathrm{F}^{+}$image on the detector in (a). (c) is obtained at $t=2.17 \mathrm{ps}$.

imaging of the dihedral angle for up to $150 \mathrm{ps,} \mathrm{which} \mathrm{goes}$ much beyond previous works where the experimental technique limited the observation times to less than 5 ps $[11,13]$.

The physical mechanism underlying torsional control by a pair of kick pulses is illustrated in Fig. 2. Figure 2(a) shows the instantaneous torsional potential, $V$, and density of the ground state wave function for the FBCBP molecule

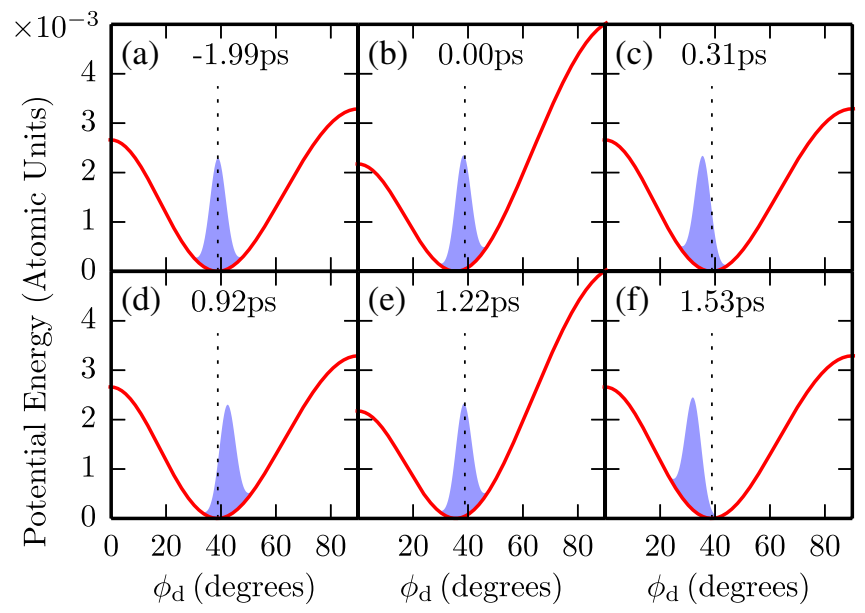

FIG. 2 (color online). Calculated time-dependent torsional potential (red line), $V$, and norm square of the wave packet (filled blue area) subject to the dynamic Stark shift from two, 200 -fs-long kick laser pulses centered at time 0.00 ps and 1.22 ps. (a) Prior to the first kick pulse. (b) During the first kick pulse. (c) Maximal displacement after the first kick pulse. (d) Shortly before the second kick pulse. (e) During the second kick pulse. (f) Maximal displacement after the second kick pulse. Dotted black lines show the center of the initial wave function. in the $0^{\circ}$ to $90^{\circ}$ range of $\phi_{d}$ prior to the kick pulses. The potential in the range $-90^{\circ}$ to $0^{\circ}$ is the mirror image of the potential presented. Through the $\phi_{d}$ dependence of the polarizability tensor, $\underline{\boldsymbol{\alpha}}$ [22], the Stark shift induced by an intense nonresonant laser pulse modifies the torsional potential while the pulse is on. For a pulse with electric field envelope $\mathbf{F}(t)$, this leads to a potential of the form

$$
V=V_{\mathrm{tor}}\left(\phi_{\mathrm{F}}-\phi_{\mathrm{Br}}\right)-\frac{1}{4} \mathbf{F}(t)^{\dagger} \underline{\underline{\boldsymbol{\alpha}}}\left(\phi_{\mathrm{F}}, \phi_{\mathrm{Br}}\right) \mathbf{F}(t),
$$

where $V_{\text {tor }}$ is the field-free torsional potential. The modification of the potential shifts its minimum and drives the molecule toward lower dihedral angles; i.e., the initial wave function is converted to a wave packet moving to the left [Fig. 2(b)]. Before the wave packet reaches the new minimum, the pulse is turned off and the wave packet continues its motion on the initial potential. This is shown in Fig. 2(c) where the wave packet has reached the maximal displacement from equilibrium. The effect of a second laser pulse depends critically on its timing with respect to both the position and velocity of the wave packet. If the second pulse arrives after one torsional period, when the wave packet is moving toward lower $\phi_{d}$ values, the wave packet will experience an additional downhill slope [Fig. 2(e)] and accelerate. As a result it moves further away from equilibrium than after the first pulse-compare Figs. 2(f) and 2(c). Alternatively, delaying the second pulse by only half a torsional period, the wave packet encounters a temporary uphill slope, strongly decreasing its torsional energy and essentially ceasing the torsional motion.

The majority of the experimental setup was accounted for earlier [13], so the description here is brief including a schematic in Fig. 1. A cold molecular beam of FBCBP seeded in helium was crossed at $90^{\circ}$ by three laser beams. The molecules were adiabatically three dimensionally (3D) aligned by a 10 ns (FWHM) nonresonant pulse from a Nd:YAG laser (YAG pulse, elliptical polarization, $I_{\text {major }} / I_{\text {minor }}=3, \lambda=1064 \mathrm{~nm}, I_{\mathrm{YAG}}=1 \times 10^{12} \mathrm{~W} \mathrm{~cm}^{-2}$ ). The torsional motion was initiated and controlled by one or two 200 fs (FWHM) laser pulses (kick pulse, linear polarization, $\lambda=800 \mathrm{~nm}, I_{\text {kick }}=3 \times 10^{13} \mathrm{~W} \mathrm{~cm}^{-2}$ ). After a variable delay the molecules were irradiated by an intense $30 \mathrm{fs}(\mathrm{FWHM}$ ) laser pulse (probe pulse, linear polarization, $\lambda=800 \mathrm{~nm}, I_{\text {probe }}=3 \times 10^{14} \mathrm{~W} \mathrm{~cm}^{-2}$ ) causing them to Coulomb explode. The cationic fragments, notably $\mathrm{Br}^{+}$and $\mathrm{F}^{+}$ions, from the Coulomb explosion were collected in a velocity map imaging system enclosing the interaction region and the velocity focused onto a position sensitive detector consisting of a microchannel plate assembly and a P47 phosphor screen. The phosphor screen was backed by a pixel imaging mass spectrometry (PIMMS) camera [23,24]

To image the torsional motion we follow the strategy from previous works $[11,13,25]$ and determine the dihedral angle directly by measuring the angle between the 
fragmenting $\mathrm{Br}^{+}$ions and $\mathrm{F}^{+}$ions. Assuming axial recoil, this is the angle between the $\mathrm{Br}$ - and $\mathrm{F}$-substituted benzene rings when the molecule is viewed along the long axis. This view is obtained by 3D alignment, induced by the YAG pulse, such that the long axis of the molecules is aligned perpendicular to the detector plane-shown in Fig. 1(a). We expect no or very little orientation of the molecule; i.e., the carbon-nitrogen $(\mathrm{CN})$ end of the FBCBP molecule (Fig. 1) is equally likely to point toward or away from the detector.

The PIMMS camera plays a crucial role in the determination of the angle between the $\mathrm{Br}^{+}$and $\mathrm{F}^{+}$ions, since it records images of all ionic species for each laser pulse sequence. This allows us to identify correlations between the emission directions of the $\mathrm{Br}^{+}$and $\mathrm{F}^{+}$ions through a covariance analysis of the velocity vectors of the recoiling ions $[25,26]$. The result of the analysis is represented as covariance images of one ion species with respect to the other. Figures 1(b) and 1(c) show examples of $\mathrm{F}^{+}$covariance images plotted relative to the $\mathrm{Br}^{+}$ion emission direction which is here arranged to be vertical. The four localized regions of intensity illustrate the relationship between the $\mathrm{Br}^{+}$and $\mathrm{F}^{+}$ion velocities and enable a direct determination of the average dihedral angle, $\left\langle\phi_{d}\right\rangle$, which is our main observable.

A very significant advantage of the new method to determine $\left\langle\phi_{d}\right\rangle$ is that no angular localization of the raw ion images is required, unlike the case in previous studies of torsion [11,13]. In those works the $\mathrm{F}^{+}$and $\mathrm{Br}^{+}$ion images were analyzed separately and an average emission angle, with respect to the kick pulse polarization, was identified for each of the two ion species such that $\left\langle\phi_{d}\right\rangle$ could be determined as the sum of the two. That analysis was only possible in the first 3-4 ps after the kick pulse where the initial 3D alignment still prevailed and, consequently, the $\mathrm{F}^{+}$and $\mathrm{Br}^{+}$ion distributions remained angularly confined. At longer times the rotation around the long molecular axis, which the kick pulse induces in addition to torsion, blurred the angular distribution of both the $\mathrm{F}^{+}$and the $\mathrm{Br}^{+}$ions to an extent that no average orientation angle of the $\mathrm{F}$ - or Br-benzene rings could be determined. The current correlation method to determine the $\left\langle\phi_{d}\right\rangle$ is insensitive to angular blurring of the ion distributions, as illustrated by the poorly resolved raw ion image for $\mathrm{F}^{+}$shown in Fig. 1(a), compared with the derived $\mathrm{F}^{+}$covariance image in Fig. 1(b). It only requires that the long axis of the molecule is kept perpendicular to the detector plane, which is ensured by the YAG pulse. As such the method should be applicable to track torsion for very long periods of time which we exploit below.

Figure 3(a) depicts $\left\langle\phi_{d}\right\rangle$ as a function of time during the first 4 ps after a single kick pulse. The experimental data show a periodic motion with an amplitude of $3^{\circ}$ and a period of $1.22 \mathrm{ps}$, determined by the torsional potential and the moments of inertia of the benzene rings. This is

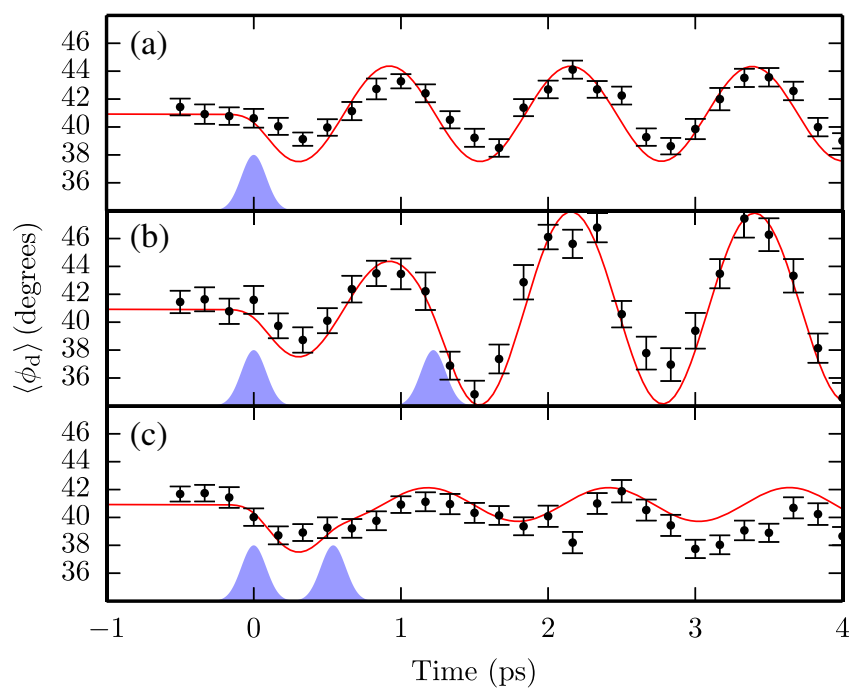

FIG. 3 (color online). The average dihedral angle as a function of time. (a) A single kick pulse. (b) Two kick pulses separated by 1.22 ps. (c) Two kick pulses separated by 0.54 ps. Black dots: experimental results. Red lines: calculated results. Blue shapes: intensity profiles of the kick pulses.

in line with the expectations based on the qualitative considerations relating to Fig. 2, in particular the initial move of $\left\langle\phi_{d}\right\rangle$ toward lower values. The observations also agree very well with the findings in Ref. [13] obtained for similar experimental parameters. Figures 3(b) and 3(c) show the $\left\langle\phi_{d}\right\rangle$ dynamics for two kick pulses. When the second pulse is applied at $1.22 \mathrm{ps}$ the amplitude of the subsequent $\left\langle\phi_{d}\right\rangle$ motion increases strongly to $6^{\circ}$, whereas it decreases to approximately $1.5^{\circ}$ when the second pulse is applied at $0.54 \mathrm{ps}$. The period of the oscillations remains essentially unchanged. These observations, demonstrating pronounced coherent control of the torsional amplitude, are also in excellent agreement with the qualitative expectations since at 1.22 ps the two benzene rings rotate toward each other [Fig. 3(a)] and the action of the second pulse should increase the amplitude. Likewise, at $0.54 \mathrm{ps}$ the two benzene rings rotate away from each other [Fig. 3(a)] and the action of the second pulse should counteract this rotation and reduce the amplitude. We expected the reduction would have been even stronger if the second pulse had been sent at $0.61 \mathrm{ps}$ (precisely half a period).

The $\left\langle\phi_{d}\right\rangle$ dynamics recorded at larger times is shown in Fig. 4. Two kick pulses separated by 1.22 ps, i.e., synchronized to yield maximal torsional amplitude were used. Because of the significant acquisition times required (limited by the $20 \mathrm{~Hz}$ repetition rate of the YAG laser) the measurements were restricted to the $0-20 \mathrm{ps}$ time window and four selected intervals up to 200 ps. Figure 4(a) shows that the amplitude gradually decreases during the first 20 ps. At 50 ps it is even smaller and at 100 ps the oscillations are hardly visible. These observations are interpreted as dispersion of the torsional wave packet 

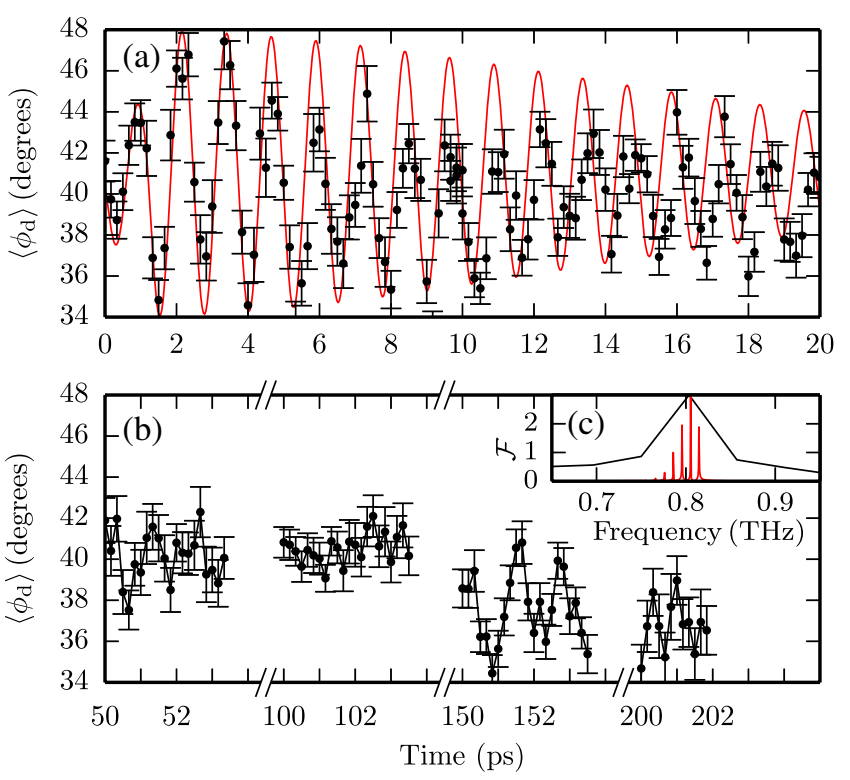

FIG. 4 (color online). The measured (black dots) and simulated (red lines) average dihedral angle as a function of time for two kick pulses separated by 1.22 ps. The inset in (c) shows the Fourier transform, $\mathcal{F}$, (arbitrary units) of $\left\langle\phi_{d}\right\rangle$ for the calculated results (red) in the interval 0-2500 ps and the experimental results (black) in the interval 0-20 ps.

due to the anharmonicity of the torsional potential [see Fig. 2(a)]. Likewise, the increased amplitude subsequently observed at $150 \mathrm{ps}$ is interpreted as revival of the torsional wave packet [27].

To rationalize our observations we calculated the torsional motion based on a further development of the models described in Refs. [11,28]. The long axis of the molecule is kept fixed and the motion of the molecule is restricted to rotation of the benzene rings around this axis. The molecule is described by a wave function on a grid of $\phi_{\mathrm{F}}$ and $\phi_{\mathrm{Br}}$. The wave function is propagated in the potential, given by Eq. (1), where $\mathbf{F}(t)$ includes the electric field from both the YAG and the kick pulses. The YAG pulse is 30 times less intense than each of the kick pulses and, therefore, the distortion of the field-free torsional potential will be 30 times smaller. As such the torsional potential including the effect from the YAG pulse [Fig. 2(a)] is essentially identical to the field-free torsional potential. The latter and the polarizability for FBCBP were computed at density functional theory level $[29,30]$ using Turbomole 6.4 [31], as described in Ref. [13].

The initial state is obtained by propagation of the timedependent Schrödinger equation in imaginary time [32] for $V_{\text {tor }}$ and the polarizability interaction of the YAG pulse. The latter interaction confines the rotation of the benzene rings corresponding to the initially $3 \mathrm{D}$ aligned molecules in the experiment. Figure 2 shows the wave packet and the torsional potential at different times during a calculation with two kick pulses. The wave packet was reduced to one dimension by integrating over lines of constant $\phi_{d}$ values and the one-dimensional representation of the potential was obtained by a cut through the two dimensional potential surface, intersecting the points of the largest amplitude of the initial wave function [28].

The calculated results are shown as the red curves in Fig. 3 and Fig. 4(a). They are shifted down by $2^{\circ}$ to match the value of $\left\langle\phi_{d}\right\rangle$ prior to the kick pulses. The agreement with the experimental findings is very good although to reproduce the absolute values of the observed oscillation amplitudes it is necessary to use an intensity of the kick pulse which is a factor of 3 lower than the experimental peak intensity. We ascribe this discrepancy mainly to averaging of the experimental signals over focal volumes where the intensity is below the peak value and partly to imperfect alignment of the long molecular axis in the experiment. The model also exhibits dispersion and revival structures similar to what was observed experimentally but the time scale of these revivals differs from the time scale in the experiment. We believe this is due to the anharmonicity of the calculated torsional potential not perfectly reproducing the anharmonicity of the real potential. Finally, Fig. 4(c) compares the Fourier transform of the first $20 \mathrm{ps}$ of the experimental data and the first $2500 \mathrm{ps}$ of the calculation. Whereas the limited time range of the experimental data prevents resolving individual components in the wave packet, the Fourier transform of the numerical data shows that approximately six torsional energy levels are significantly populated by the kick pulses.

Our results point to new opportunities. First, the current results show that it is possible to localize energy in the lowest vibrational mode [11] of a complex molecule and follow the dynamics of the associated torsional wave packet for extended periods of time. By increasing the initial torsional energy, through employing more kick pulses, it is likely that intramolecular vibrational relaxation (IVR) becomes important. Changes to the pronounced observed oscillations in $\left\langle\phi_{d}\right\rangle$ beyond those resulting from wave packet dispersion provide a sensitive, direct, and ultrafast probe of IVR. Second, our calculation shows that the amplitude of the oscillations in $\left\langle\phi_{d}\right\rangle$ increases almost linearly with the number of kick pulses until the wave packet gains enough energy to cross the torsional barrier at $\phi_{d}=0$. For pulse durations identical to those used in the calculation presented in Fig. 3, it occurs after eight pulses. Such a pulse sequence is experimentally feasible [33] and will create a molecule which periodically switches between the two enantiomeric forms of the axially chiral FBCBP. The central reaction coordinate, $\left\langle\phi_{d}\right\rangle$, during the isomerization can be imaged with the method presented here. Third, it should be possible to conduct the current experiment on molecules embedded in helium nanodroplets [34] which would enable studies of how a dissipative environment influences the torsional dynamics [35]. 
In conclusion, we have demonstrated that torsion of a substituted biphenyl molecule can be coherently controlled by a pair of strong, nonresonant fs laser pulses. When the pulses are separated by a torsional period the torsional amplitude almost doubles compared to the result for a single pulse. Our theoretical analysis rationalizes the experimental findings and shows that additional, correctly timed, pulses will further enhance torsion. In combination with the novel ability to image the dihedral angle for extended periods of time, our results point to new avenues for research on intramolecular vibrational relaxation and enantiomeric transformations in complex molecules, isolated or coupled to a dissipative environment.

L. B. M. acknowledges ERC-StG (Project No. 277767TDMET) and the VKR Centre of Excellence QUSCOPE. H.S. acknowledges ERC-AdG (Project No. 320459DropletControl), the Lundbeck Foundation, the Carlsberg Foundation, and the Danish Council for Independent Research (Natural Sciences). M. J. acknowledges that CSC - the Finnish IT Center for Science hosted parts of the calculations. M. B. acknowledges the support of EPSRC via Programme Grant No. EP/L005913/1, the EU through Grant No. FP7 ITN "ICONIC" (Project Grant No. 238671), STFC through a PNPAS award and a mini-IPS grant (ST/J002895/1), and a proof of concept grant from ISIS Innovation, Ltd. A. L. thanks the DFG for support via Grant No. La 3209/1-1.

[1] A. M. Weiner, Rev. Sci. Instrum. 71, 1929 (2000).

[2] T. Brixner and G. Gerber, Opt. Lett. 26, 557 (2001).

[3] H. Rabitz, R. de Vivie-Riedle, M. Motzkus, and K. Kompa, Science 288, 824 (2000).

[4] T. Brixner and G. Gerber, ChemPhysChem 4, 418 (2003).

[5] D. Townsend, B. J. Sussman, and A. Stolow, J. Phys. Chem. A 115, 357 (2011).

[6] F. Rosca-Pruna and M. J. J. Vrakking, Phys. Rev. Lett. 87, 153902 (2001).

[7] H. Sakai, C. P. Safvan, J. J. Larsen, K. M. Hilligsøe, K. Hald, and H. Stapelfeldt, J. Chem. Phys. 110, 10235 (1999).

[8] H. Niikura, P. B. Corkum, and D. M. Villeneuve, Phys. Rev. Lett. 90, 203601 (2003).

[9] H. Niikura, D. M. Villeneuve, and P. B. Corkum, Phys. Rev. A 73, 021402 (2006).

[10] S. Ramakrishna and T. Seideman, Phys. Rev. Lett. 99, 103001 (2007).

[11] C. B. Madsen, L. B. Madsen, S. S. Viftrup, M. P. Johansson, T. B. Poulsen, L. Holmegaard, V. Kumarappan, K. A. Jørgensen, and H. Stapelfeldt, Phys. Rev. Lett. 102, 073007 (2009).

[12] L. H. Coudert, L. F. Pacios, and J. Ortigoso, Phys. Rev. Lett. 107, 113004 (2011).
[13] J. L. Hansen, J. H. Nielsen, C. B. Madsen, A. T. Lindhardt, M. P. Johansson, T. Skrydstrup, L. B. Madsen, and H. Stapelfeldt, J. Chem. Phys. 136, 204310 (2012).

[14] K. F. Reim, J. Nunn, V. O. Lorenz, B. J. Sussman, K. C. Lee, N. K. Langford, D. Jaksch, and I. A. Walmsley, Nat. Photonics 4, 218 (2010).

[15] H. Stapelfeldt, H. Sakai, E. Constant, and P. B. Corkum, Phys. Rev. Lett. 79, 2787 (1997).

[16] R. Fulton, A. I. Bishop, M. N. Shneider, and P. F. Barker, Nat. Phys. 2, 465 (2006).

[17] B. J. Sussman, D. Townsend, M. Y. Ivanov, and A. Stolow, Science 314, 278 (2006).

[18] M. Leibscher, I. S. Averbukh, and H. Rabitz, Phys. Rev. Lett. 90, 213001 (2003).

[19] C. Z. Bisgaard, M. D. Poulsen, E. Péronne, S. S. Viftrup, and H. Stapelfeldt, Phys. Rev. Lett. 92, 173004 (2004).

[20] K. F. Lee, I. V. Litvinyuk, P. W. Dooley, M. Spanner, D. M. Villeneuve, and P. B. Corkum, J. Phys. B 37, L43 (2004).

[21] K. F. Lee, E. A. Shapiro, D. M. Villeneuve, and P. B. Corkum, Phys. Rev. A 73, 033403 (2006).

[22] C. B. Madsen, L. B. Madsen, S. S. Viftrup, M. P. Johansson, T. B. Poulsen, L. Holmegaard, V. Kumarappan, K. A. Jørgensen, and H. Stapelfeldt, J. Chem. Phys. 130, 234310 (2009).

[23] A. Nomerotski, M. Brouard, E. Campbell, A. Clark, J. Crooks, J. Fopma, J. J. John, A. J. Johnsen, C. Slater, R. Turchetta, C. Vallance, E. Wilman, and W. H. Yuen, JINST 5, C07007 (2010).

[24] J. J. John, M. Brouard, A. Clark, J. Crooks, E. Halford, L. Hill, J. W. L. Lee, A. Nomerotski, R. Pisarczyk, I. Sedgwick, C. S. Slater, R. Turchetta, C. Vallance, E. Wilman, B. Winter, and W. H. Yuen, JINST 7, C08001 (2012).

[25] C. S. Slater, S. Blake, M. Brouard, A. Lauer, C. Vallance, J. J. John, R. Turchetta, A. Nomerotski, L. Christensen, J. H. Nielsen, M. P. Johansson, and H. Stapelfeldt, Phys. Rev. A 89, 011401 (2014).

[26] L. J. Frasinski, K. Codling, and P. A. Hatherly, Science 246, 1029 (1989).

[27] B. A. Ashwell, S. Ramakrishna, and T. Seideman, J. Chem. Phys. 138, 044310 (2013).

[28] C. B. Brandt, Master's thesis, Aarhus University, 2012.

[29] A. D. Becke, J. Chem. Phys. 98, 5648 (1993).

[30] C. Lee, W. Yang, and R. G. Parr, Phys. Rev. B 37, 785 (1988).

[31] R. Ahlrichs, M. Bär, M. Häser, H. Horn, and C. Kölmel, Chem. Phys. Lett. 162, 165 (1989).

[32] R. Kosloff and H. Tal-Ezer, Chem. Phys. Lett. 127, 223 (1986).

[33] J. P. Cryan, P. H. Bucksbaum, and R. N. Coffee, Phys. Rev. A 80, 063412 (2009).

[34] D. Pentlehner, J. H. Nielsen, A. Slenczka, K. Mølmer, and H. Stapelfeldt, Phys. Rev. Lett. 110, 093002 (2013).

[35] B. A. Ashwell, S. Ramakrishna, and T. Seideman, J. Phys. Chem. C 117, 22391 (2013). 